\title{
Article
}

\section{Solving the electrical control of magnetic coercive field paradox}

\author{
Vopson, M. M. and Lepadatu, S. \\ Available at https://clok.uclan.ac.uk/11792/ \\ Vopson, M. M. and Lepadatu, S. orcid iconORCID: 0000-0001-6221-9727 \\ (2014) Solving the electrical control of magnetic coercive field paradox. \\ Applied Physics Letters, 105 (12). p. 122901. ISSN 0003-6951
}

It is advisable to refer to the publisher's version if you intend to cite from the work. http://dx.doi.org/10.1063/1.4896521

For more information about UCLan's research in this area go to

http://www.uclan.ac.uk/researchgroups/ and search for <name of research Group>.

For information about Research generally at UCLan please go to http://www.uclan.ac.uk/research/

All outputs in CLoK are protected by Intellectual Property Rights law, including Copyright law. Copyright, IPR and Moral Rights for the works on this site are retained by the individual authors and/or other copyright owners. Terms and conditions for use of this material are defined in the policies page.

\section{CLoK}

Central Lancashire online Knowledge www.clok.uclan.ac.uk

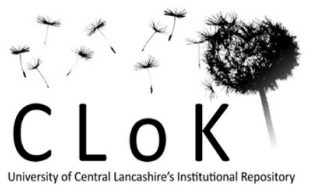




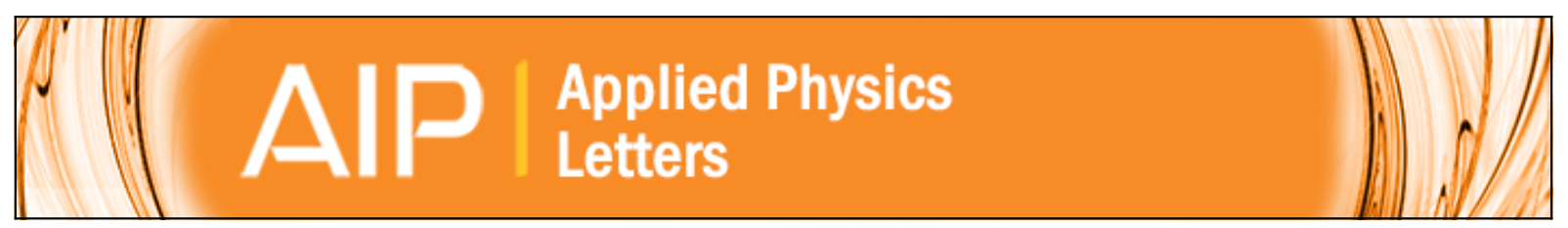

\section{Solving the electrical control of magnetic coercive field paradox}

M. M. Vopson and S. Lepadatu

Citation: Applied Physics Letters 105, 122901 (2014); doi: 10.1063/1.4896521

View online: http://dx.doi.org/10.1063/1.4896521

View Table of Contents: http://scitation.aip.org/content/aip/journal/apl/105/12?ver=pdfcov

Published by the AIP Publishing

\section{Articles you may be interested in}

0.7BiFeO3-0.3BaTiO3-Y3Fe5O12 composites with simultaneously improved electrical and magnetic properties

J. Appl. Phys. 111, 024104 (2012); 10.1063/1.3677944

Tunable fringe magnetic fields induced by converse magnetoelectric coupling in a FeGa/PMN-PT multiferroic heterostructure

J. Appl. Phys. 110, 123916 (2011); 10.1063/1.3672822

Switchable voltage control of the magnetic coercive field via magnetoelectric effect

J. Appl. Phys. 110, 043919 (2011); 10.1063/1.3626748

Voltage control of the magnetic coercive field: Multiferroic coupling or artifact?

J. Appl. Phys. 109, 066101 (2011); 10.1063/1.3558982

Electrically controlled magnetization switching in a multiferroic heterostructure

Appl. Phys. Lett. 97, 052502 (2010); 10.1063/1.3475417

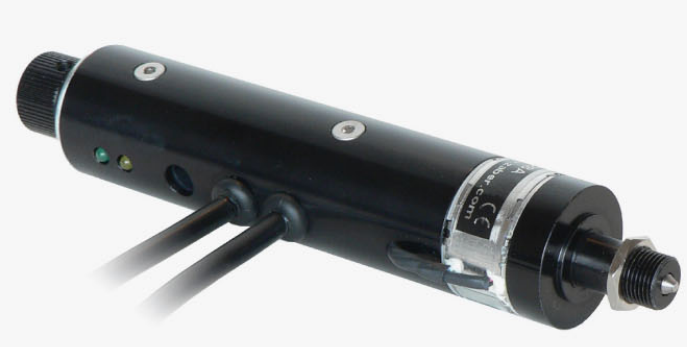

Automate your set-up with 


\title{
Solving the electrical control of magnetic coercive field paradox
}

\author{
M. M. Vopson ${ }^{1, a)}$ and S. Lepadatu ${ }^{2}$ \\ ${ }^{1}$ Faculty of Science, SEES, University of Portsmouth, Portsmouth PO1 3QL, United Kingdom \\ ${ }^{2}$ National Physical Laboratory, Functional Materials Group, Teddington TW11 0LW, United Kingdom
}

(Received 20 August 2014; accepted 13 September 2014; published online 22 September 2014)

\begin{abstract}
The ability to tune magnetic properties of solids via electric voltages instead of external magnetic fields is a physics curiosity of great scientific and technological importance. Today, there is strong published experimental evidence of electrical control of magnetic coercive fields in composite multiferroic solids. Unfortunately, the literature indicates highly contradictory results. In some studies, an applied voltage increases the magnetic coercive field and in other studies the applied voltage decreases the coercive field of composite multiferroics. Here, we provide an elegant explanation to this paradox and we demonstrate why all reported results are in fact correct. It is shown that for a given polarity of the applied voltage, the magnetic coercive field depends on the sign of two tensor components of the multiferroic solid: magnetostrictive and piezoelectric coefficient. For a negative applied voltage, the magnetic coercive field decreases when the two material parameters have the same sign and increases when they have opposite signs, respectively. The effect of the material parameters is reversed when the same multiferroic solid is subjected to a positive applied voltage. (C) 2014 AIP Publishing LLC. [http://dx.doi.org/10.1063/1.4896521]
\end{abstract}

Electrical control of magnetism in solids is an interesting field of research, which has its basis on the magnetoelectric effect in multiferroic materials. Today, there are encouraging reports of various experimental studies showing successful measurements of the electrical induced magnetization change, ${ }^{1,2}$ switching of the exchange bias field in ferromagnet/anti-ferromagnet structures via a voltage ${ }^{3,4}$ and electrical tuning of magnetism in hybrid spintronics/multiferroic composites designed for advanced random access memories or logic operations. ${ }^{5-8}$

Due to the huge versatility offered by the interplay between electric and magnetic properties in solids, magnetoelectric effect of multiferroics is currently of great interest for fundamental studies as well as technological applications. ${ }^{9-19}$ In particular, the ability to control magnetic coercive field through an electric voltage (field) offers unprecedented advantages in spin-electronic devices and magnetic data storage technologies. The prospect of novel applications has stimulated a massive surge in research of multiferroic materials, especially composites. ${ }^{20-22}$ Experiments of voltage manipulation of magnetic coercive field in ferroelectric/ferromagnetic composite multiferroics have been widely reported.,23-26 However, a closer examination of the literature reveals that the results are in fact highly contradictory. In some studies, it has been reported that an applied voltage (electric field) to a composite multiferroic would increase its magnetic coercive field, while in other studies the same process had the opposite effect. Table I shows a summary of some of these contradictory studies.

Giving the critical importance of the electric control of magnetic coercive field for technical applications, the normal question one would ask is: "Which of these results are correct?" Answering this question and providing a clear

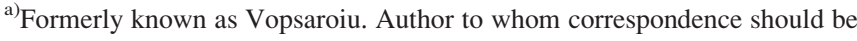
addressed. Electronic mail: melvin.vopson@port.ac.uk.
}

understanding of the governing physics would greatly help future studies and the design of novel applications based on these interesting materials. In this article, we provide an explanation to this apparent paradox. We will show that, in fact, all reported studies are correct. The apparent contradictory responses of the magnetic coercive field to an electrical excitation in various composite multiferroics are related to the constitutive material parameters within the corresponding composite structures, as detailed below.

Let us assume a multiferroic bi-layer composite structure consisting of a polycrystalline magnetic thin film and a piezo-ferroelectric layer. The magnetic thin film is made up of individual magnetic grains, with each grain being considered uni-axial magnetic single domain. Such magnetic entities can be well described in the Stoner-Wohlfarth (S-W) formalism. We further impose grains separation distance large enough to ensure that no exchange or magneto-static inter-grain interactions are present. This approximation is indeed valid for magnetic data storage thin film technology and allows the analysis of an individual constituent particle to provide a detailed insight into the overall magnetic reversal mechanism governing the entire sample.

Figure 1(a) shows the schematic diagram of the composite multiferroic bi-layer system, which is expected to display the so called electrically induced strain-mediated magnetoelectric effect. This effect is defined as the modification of

TABLE I. Experimental reported magnetic coercive field variation with an applied voltage for various bi-layer composite multiferroic structures.

\begin{tabular}{lcc}
\hline Composite multiferroic & Coercive field trend vs applied voltage & References \\
\hline $\mathrm{Fe} / \mathrm{BaTiO}$ & Decrease & 24 \\
$\mathrm{FeBSiC} / \mathrm{PZT}$ & Increase & 2 \\
$\mathrm{Co}_{50} \mathrm{Fe}_{50} / \mathrm{PZT}$ & Increase & 25 \\
$\mathrm{NiFe}_{\mathrm{BaTiO}}$ & Decrease & 23 \\
$\mathrm{CoFe} / \mathrm{PZT}$ & Decrease and increase & 26 \\
\hline \hline
\end{tabular}




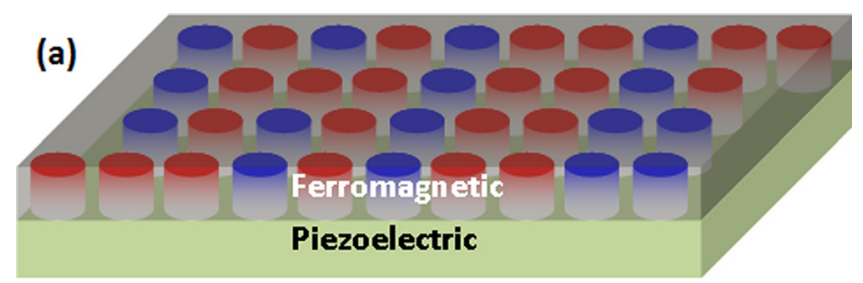

(b)

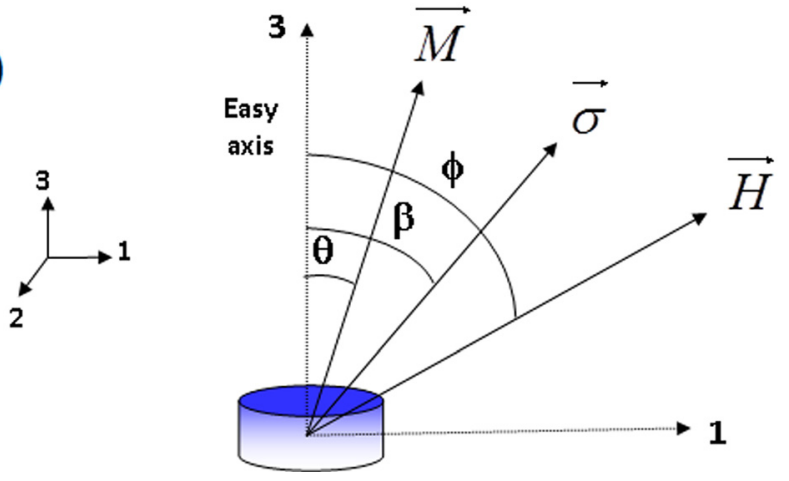

FIG. 1. (a) Schematic of multiferroic composite by-layer system consisting of a polycrystalline ferromagnetic thin film strain-mediated coupled to a piezo-electric layer. (b) Uni-axial single domain magnetic particle under applied $\mathrm{H}$ field and $\sigma$ stress.

the magnetic properties (coercive field and/or magnetization) of the magnetic layer within the composite structure as a response to an electrical excitation (voltage/electric field) to the piezo-layer. The effect is widely accepted to occur due to the applied stress which results from the deformation transferred to the magnetic grains by the piezo-substrate when electrically activated by the application of an electric field/voltage.

If $\varphi$ is the angle between the applied field and the magnetic easy axis (EA) of a magnetic particle, $\beta$ is the angle between the applied external stress and the EA, and $\theta$ is the angle between the magnetization and the EA (see Fig. 1(b)), then for the ith magnetic particle of volume $V_{i}$, the total energy per unit volume under applied magnetic field and external stress is

$$
\begin{aligned}
E_{t o t} / V_{i}= & K_{a} \sin ^{2} \theta_{i}-\mu_{0} H M_{S} \cos \left(\varphi-\theta_{i}\right) \\
& -\frac{3}{2} \lambda_{s} \sigma_{1} \sin ^{2}\left(\beta-\theta_{i}\right),
\end{aligned}
$$

where the first term represents the magneto-crystalline anisotropy energy and $\mathrm{K}_{\mathrm{a}}$ is the anisotropy constant; the second term represents the magnetic potential energy due to the applied $\mathrm{H}$ magnetic field and $\mathrm{M}_{\mathrm{s}}$ is the saturation magnetization; and the third term is the magneto-elastic energy for a uni-axial particle where $\lambda_{\mathrm{s}}$ is the saturation magnetostriction coefficient.

Assuming that the ferroelectric substrate is poled in the 3 direction (out of plane), a stress will result in the plane of the magnetic film due to $\mathrm{d}_{31}$ piezo-electric coupling, which is given by $\sigma_{1}=\mathrm{Yd}_{31} \mathrm{E}_{3}=\mathrm{Yd}_{31} \mathrm{~V}_{3} / \mathrm{t}_{\mathrm{e}}$, where $\mathrm{t}_{\mathrm{e}}$ is the thickness of the substrate, $d_{31}$ piezo-electric coefficient, $Y$ is the Young's modulus of the ferromagnetic grain, and $V_{3}$ is the amplitude of the applied voltage.

We now impose the stress angle along 1 axis $(\beta=\pi / 2)$ and the external magnetic applied field at an angle $\pi$ to the easy axis in order to produce its reversal $(\varphi=\pi)$.
With these restrictions imposed, we determine the equilibrium position of the magnetization by minimizing the total energy in respect with the magnetization angle $\left(\theta_{\mathrm{i}}\right)$ using the condition $\mathrm{dE}_{\mathrm{tot}} / \mathrm{d} \theta_{\mathrm{i}}=0$. From this we determine the magnetic anisotropy field as the field required to produce, at equilibrium, a $\pi$ rotation of the $\mathrm{M}_{\mathrm{s}}$ from the EA direction. The following relation for the magnetic anisotropy field is obtained:

$$
H_{a}=\frac{1}{\mu_{0} M_{s}} \cdot\left(2 K_{a} \pm \frac{3 \lambda_{s} Y d_{31}\left|V_{3}\right|}{t_{e}}\right) .
$$

For zero applied stress $\left(\sigma_{1}=\mathrm{Yd}_{31} \mathrm{~V}_{3} / \mathrm{t}_{\mathrm{e}}=0\right.$, i.e., $\left.\mathrm{V}_{3}=0\right)$, the anisotropy field is given by the well-known StonerWohlfarth relation: $\mathrm{H}_{\mathrm{a}}=2 \mathrm{~K}_{\mathrm{a}} / \mu_{0} \mathrm{M}_{\mathrm{s}}$. However, in the case of an active piezo-substrate inflicting a longitudinal strain to the magnetic grains, we expect a change of the magnetic anisotropy field when $\mathrm{V}_{3} \neq 0$.

According to relation (2), the effective anisotropy field can be either increased or decreased depending on the constituent material parameters dictating the signs of $\lambda_{\mathrm{s}}$ and $d_{31}$. The \pm in relation (2) indicates the fact that the polarity of the applied voltage can be positive or negative resulting in compressive or tensile stress with direct consequences on the effective magnetic anisotropy field (i.e., coercive field).

Accordingly, a positive applied electric field/voltage $( \pm \rightarrow+$ in relation (2)) will result in a reduction of the anisotropy field when magnetostriction and piezoelectric coefficient have opposite signs and an increase in the anisotropy field when the two parameters have the same signs (both either negative or positive). Similarly, a negative applied electric field/voltage $( \pm \rightarrow-$ in relation (2)) will result in a reverse effect: the reduction of the anisotropy field when magnetostriction and piezoelectric coefficients have the same signs and an increase in the anisotropy field when the two parameters have opposite signs. Since the magnetic coercive field is directly proportional to the magnetic anisotropy field as $\mathrm{H}_{\mathrm{c}}=\mathrm{H}_{\mathrm{a}} \cdot \mathrm{f}\left(\mathrm{K}_{\mathrm{a}}, \mathrm{t}, \mathrm{T}\right),{ }^{27}$ where $\mathrm{f}$ is a function of magneto-crystalline anisotropy constant, time, and temperature, then variations in the magnetic anisotropy field would also result in similar variations of the magnetic coercive field. The propagation of material parametric signs into the magnetic anisotropy and coercive field is represented in Table II.

In the present model, we assumed multiferroic composites with "out-of-plane" magnetic anisotropy, because this is the standard format currently used in magnetic data storage devices and this is also the most likely sector where applications of this research could emerge. However, the present theory is generally applicable and the resulting equations are invariant to the "in-plane"/"out-of-plane" orientation of the

TABLE II. The expected effective magnetic anisotropy and coercive field variation with the applied voltage as a function of the sign of the magnetostriction and piezoelectric coefficients for positive and negative applied voltage polarities. $\uparrow=\mathrm{H}_{\mathrm{a}} / \mathrm{H}_{\mathrm{c}}$ goes up and $\downarrow=\mathrm{H}_{\mathrm{a}} / \mathrm{H}_{\mathrm{c}}$ goes down.

\begin{tabular}{lllllllll}
\hline \hline Sign of $\mathbf{V}_{\mathbf{3}}$ & \multicolumn{3}{c}{ Positive } & & \multicolumn{3}{c}{ Negative } \\
\hline Sign of $\mathbf{d}_{\mathbf{3 1}}$ & + & - & + & - & + & - & + & - \\
Sign of $\boldsymbol{\lambda}_{\mathbf{s}}$ & + & - & - & + & + & - & - & + \\
Coercive field $\mathbf{H}_{\mathbf{a}} / \mathbf{H}_{\mathbf{c}}$ & $\uparrow$ & $\uparrow$ & $\downarrow$ & $\downarrow$ & $\downarrow$ & $\downarrow$ & $\uparrow$ & $\uparrow$ \\
\hline \hline
\end{tabular}


magnetic easy axis, providing the induced stress is at $\beta=\pi / 2$ angle to the easy axis and the magnetic field is applied at $\phi=\pi$ to the easy axis to produce magnetization reversal.

We now refer to the experimental results summarized in Table I. Both $\mathrm{BaTiO}_{3}$ and $\mathrm{PbZrTiO}_{3}$ (PZT) piezoferroelectric components have negative $d_{31}$ piezo-electric coefficients. Typical value of $\mathrm{d}_{31}$ in the case of PZT is $\mathrm{d}_{31}=-1.7 \times 10^{-10} \mathrm{~m} / \mathrm{V}$, while in the case of $\mathrm{BaTiO}_{3}$ is $\mathrm{d}_{31}=-58 \times 10^{-12} \mathrm{~m} / \mathrm{V} .^{28}$

The magnetic components $\mathrm{Fe}$ and NiFe have both negative saturation magnetostriction coefficients $\left(\lambda^{\mathrm{Fe}}{ }_{\mathrm{s}}=-0.8 \times 10^{-5}\right.$ (Ref. 29) and $\lambda^{\mathrm{NiFe}}{ }_{\mathrm{s}}=-0.15 \times 10^{-5}$ (Ref. 30)), while CoFe and $\mathrm{FeBSiC}$ are characterized by positive magnetostriction coefficients $\left(\lambda^{\mathrm{CoFe}}=6.5 \times 10^{-5}\right.$ (Ref. 31) and $\lambda^{\mathrm{FeBSiC}}{ }_{\mathrm{s}}=$ $4 \times 10^{-5}$ (Ref. 32)). Since $d_{31}<0$ in all present cases and according to the selection rules listed in Table II, we deduce that for a negative applied voltage we expect the magnetic coercive field to go down for $\mathrm{FeBSiC} / \mathrm{PZT}$ and $\mathrm{Co}_{50} \mathrm{Fe}_{50} / \mathrm{PZT}$ and to go up for $\mathrm{Fe} / \mathrm{BaTiO}_{3}$ and $\mathrm{NiFe} / \mathrm{BaTiO}_{3}$ multiferroic composites. This is exactly what we observed for all composite multiferroics listed in Table I. Reversing the polarity of the applied voltage is expected to reverse the trends in the coercive fields/ effective anisotropy. However, not all experimental studies reported the reversal of the coercive field trend upon changing the polarity of the applied electric field/voltage.

We believe that this is related to the fact that Eqs. (1) and (2) are only applicable in the linear strain approximation (i.e., $\mathrm{x}_{1}=\mathrm{d}_{31} \mathrm{E}_{3}$ ). It is important to note that strain-E field curves for applied electric fields larger than the electric coercive field display a highly non-linear hysteretic behavior (classical butterfly strain-E field loops). For applied electric fields larger than the coercive field, the strain has the same sign regardless of the applied electric field polarity.

The predicted selection rules listed in Table II are fully confirmed experimentally in Ref. 26, where Boukari et al. report experimental studies of $\mathrm{CoFe} / \mathrm{PZT}$ composite multiferroic. Since $\lambda^{\mathrm{CoFe}}{ }_{\mathrm{s}}>0$ and $\mathrm{d}^{\mathrm{PZT}}{ }_{31}<0$, according to our theory, it is expected that an applied positive voltage would decrease the magnetic coercive field and an applied negative voltage would increase the magnetic coercive field, which is exactly what has been reported by Boukari et al. ${ }^{26}$

In order to test the theory, we simulated the magnetic hysteresis loops of two multiferroic composite systems consisting of piezoelectric PZT/Ferromagnet. Hysteresis loops for these systems were calculated using the Landau-LifshitzGilbert equation $(3)^{33}$ applied to a single-domain particle, i.e., the Stoner-Wohlfarth model of Eq. (1)

$$
\frac{\partial M}{\partial t}=-\gamma M \times H_{e f f}+\frac{\alpha}{M_{S}} M \times \frac{\partial M}{\partial t} .
$$

Here, $\mathrm{M}$ is the magnetization vector of the single-domain particle with saturation magnetization $\mathrm{M}_{\mathrm{S}}$ taken as $\mathrm{M}_{\mathrm{S}}=10^{6} \mathrm{~A} / \mathrm{m}$. $\alpha$ is the Gilbert damping taken as $\alpha=0.005$, $\gamma=2.21 \times 10^{5} \mathrm{~m} / \mathrm{A} \mathrm{s}$ is the gyromagnetic ratio, and $\mathrm{H}_{\mathrm{eff}}$ is the total magnetic field including contributions from the applied field and anisotropy field of Eq. (2). Equation (3) was integrated numerically using the 2nd order AdamsBashforth-Moulton method. ${ }^{34}$ For each applied magnetic field step, the relaxed magnetization was obtained under the
TABLE III. Computation parameters of two magnetic materials with positive and negative magnetostriction coefficients, respectively.

\begin{tabular}{lcccc}
\hline \hline Material & $\lambda_{\mathrm{s}}$ & $\mathrm{K}_{\mathrm{a}}\left(\mathrm{J} / \mathrm{m}^{3}\right)$ & $\mathrm{Y}(\mathrm{GPa})$ & References \\
\hline $\mathrm{Ni}_{84} \mathrm{Fe}_{16}$ & $-0.5 \times 10^{-5}$ & $0.8 \times 10^{3}$ & 180 & 30 \\
$\mathrm{Co}_{50} \mathrm{Fe}_{50}$ & $+6.5 \times 10^{-5}$ & $1.5 \times 10^{4}$ & 200 & 31 \\
\hline \hline
\end{tabular}

stopping condition $\left|\mathrm{M} \times \mathrm{H}_{\mathrm{eff}}\right| / \mathrm{M}_{\mathrm{S}}<10^{-5}$. Within the Brown formalism, ${ }^{35}$ the magnetization is in the equilibrium configuration for $\mathrm{M} \times \mathrm{H}_{\mathrm{eff}}=0$. Numerically, as we have checked, the equilibrium condition is reached before the stopping threshold used here.

As already mentioned, PZT has typically negative $\mathrm{d}_{31}$ coefficient. We therefore chose for our simulations one ferromagnetic material with positive and one with negative saturation magnetostriction coefficient. The magnetic materials chosen for the purpose of computational testing of this concept are $\mathrm{Co}_{50} \mathrm{Fe}_{50}$ and $\mathrm{Ni}_{84} \mathrm{Fe}_{16}$.

Table III shows the magnetic parameters used in our computations. In addition, we imposed that the PZT layer is $\mathrm{t}_{\mathrm{e}}=500 \mathrm{~nm}$ thick and has a piezoelectric coefficient of $\mathrm{d}_{31}=-1.7 \times 10^{-10} \mathrm{~m} / \mathrm{V}$.

Magnetic hysteresis loops for different applied voltage amplitudes and polarities were generated using the model described above with the magnetic field applied at a $5^{\circ}$ angle to the uniaxial anisotropy axis and the magnetization component plotted along the applied magnetic field direction.

Figure 2 shows the magnetic hysteresis loops of the $\mathrm{Co}_{50} \mathrm{Fe}_{50} / \mathrm{PZT}$ composite multiferroic simulated for applied voltages of $\mathrm{V}_{3}=0, \pm 0.5$, and $\pm 1.5 \mathrm{~V}$. Since $\lambda^{\mathrm{CoFe}}{ }_{\mathrm{s}}>0$ and $\mathrm{d}^{\mathrm{PZT}}{ }_{31}<0$, then a decrease in the coercive field at positive applied voltages is observed, as predicted by our theory. When the polarity of the applied electric field is reversed, the magnetic coercive field increases exactly as predicted by the selection rules given in Table II.

Note for $\mathrm{V}_{3}=0 \mathrm{~V}$, the coercive field predicted by Eq. (2) is obtained as $\mu_{0} \mathrm{H}_{\mathrm{C}}=23 \mathrm{mT}$. Magnetic coercive field of $\mathrm{CoFe}$ in relaxed state can be increased/decreased by as much as $40 \%$ under applied voltage of $1.5 \mathrm{~V}$.

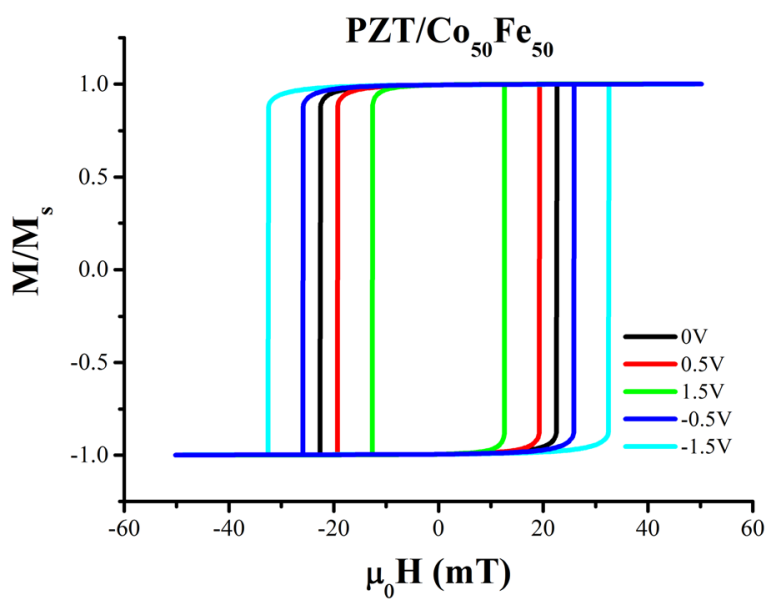

FIG. 2. Magnetic hysteresis loops of CoFe/PZT multiferroic composite under electrical excitation. Simulations performed using S-W magnetization reversal model. 


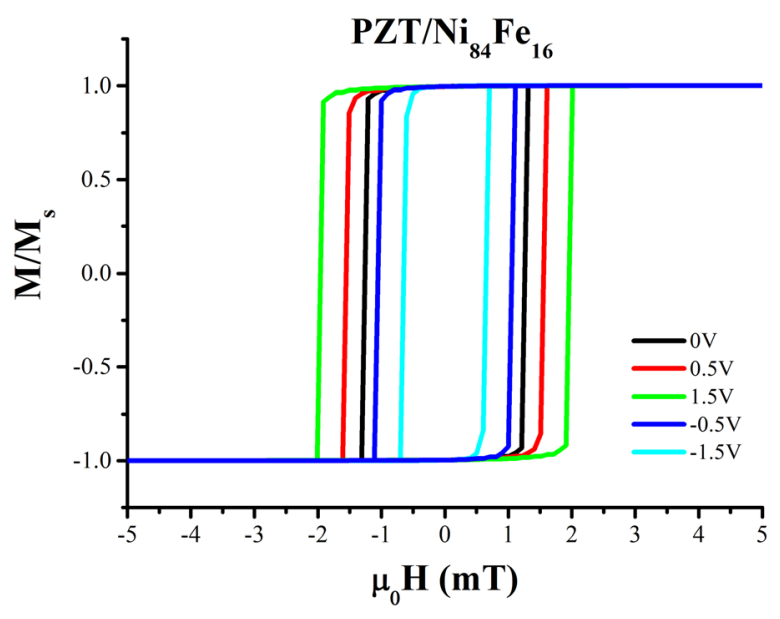

FIG. 3. Magnetic hysteresis loops of NiFe/PZT multiferroic composite under electrical excitation. Simulations performed using S-W magnetization reversal model.

Figure 3 shows the same data calculated for the $\mathrm{Ni}_{84} \mathrm{Fe}_{16} / \mathrm{PZT}$ multiferroic system under the excitation of $\mathrm{V}_{3}=0$, \pm 0.5 , and $\pm 1.5 \mathrm{~V}$. Since $\lambda_{\mathrm{s}}^{\mathrm{NiFe}}<0$ and $\mathrm{d}^{\mathrm{PZT}}{ }_{31}<0$, this time a $50 \%$ decrease in the magnetic coercive as a function of the applied voltage is observed when $-1.5 \mathrm{~V}$ is applied and an increase of $50 \%$ when $+1.5 \mathrm{~V}$ is applied. Again, note that the coercive field for $\mathrm{V}_{3}=0 \mathrm{~V}$ predicted by Eq. (2) is correctly reproduced as $\mu_{0} \mathrm{H}_{\mathrm{C}}=1.3 \mathrm{mT}$.

In conclusion, we clarified the paradox of the electric control of the magnetic coercive field in multiferroic composites which was characterized by highly confusing and contradictory experimental results.

A full understanding of the governing physics behind this effect is critical for the design of suitable multiferroic samples and devices. We demonstrated that the strain mediated magneto-electric coupling ${ }^{36,37}$ significantly alters the effective magneto-crystalline anisotropy field of magnetic grains when they are coupled to a piezo-electric active material.

A formula describing the effective magneto-crystalline anisotropy field of a multiferroic coupled magnetic bi-layer system has been derived. The theory predicts that for a given polarity of the applied electric field, the sign of $\lambda_{\mathrm{s}}$ and $d_{31}$ parameters determine the variation of the effective magnetic anisotropy/coercive field with the applied voltage. This research is critical for future studies and device applications of composite multiferroics where electrical control of magnetic properties is required.

${ }^{1}$ C. Thiele, K. Dorr, O. Bilani, J. Rodel, and L. Schultz, Phys. Rev. B 75, 054408 (2007).

${ }^{2}$ J. Ma, Y. Lin, and C. W. Nan, J. Phys. D: Appl. Phys. 43, 012001 (2010).

${ }^{3}$ X. He, Y. Wang, N. Wu, A. N. Caruso, E. Vescovo, K. D. Belashchenko, P. A. Dowben, and C. Binek, Nat. Mater. 9, 579 (2010).

${ }^{4}$ P. Borisov, A. Hochstrat, X. Chen, W. Kleemann, and C. Binek, Phys. Rev. Lett. 94, 117203 (2005).
${ }^{5}$ X. Chen, A. Hochstrat, P. Borisov, and W. Kleemann, Appl. Phys. Lett. 89, 202508 (2006).

${ }^{6}$ D. Chiba, F. Matsukura, and H. Ohno, Appl. Phys. Lett. 89, 162505 (2006).

${ }^{7}$ J. M. Hu, Z. Li, J. Wang, and C. W. Nan, J. Appl. Phys. 107, 093912 (2010).

${ }^{8}$ I. Stolichnov, S. W. E. Reister, H. J. Trodahl, N. Setter, A. W. Rushforth, K. W. Edmonds, R. P. Campion, C. T. Foxton, B. L. Gallagher, and T. Jungwirth, Nat. Mater. 7, 464-467 (2008).

${ }^{9}$ C. Kirchhof, M. Krantz, I. Teliban, R. Jahns, S. Marauska, B. Wagner, R. Knochel, M. Gerken, D. Mayners, and E. Quandt, Appl. Phys. Lett. 102(23), 232905 (2013).

${ }^{10}$ R. Jahns, A. Piorra, E. Lage, C. Kirchhof, D. Mayners, J. L. Gugat, M. Krantz, M. Gerken, R. Knochel, and E. Quandt, J. Am. Ceram. Soc. 96(6), 1673-1681 (2013).

${ }^{11}$ S. X. Dong, J. Y. Zhai, F. Bai, J.-F. Li, and D. Viehland, Appl. Phys. Lett. 87, 062502 (2005).

${ }^{12}$ U. Laletsin, N. Padubnaya, G. Srinivasan, and C. P. Devreugd, Appl. Phys. A: Mater. Sci. Process. 78, 33 (2004).

${ }^{13}$ Y. K. Fetisov and G. Srinivasan, Appl. Phys. Lett. 88, 143503 (2006).

${ }^{14}$ M. Vopsaroiu, J. Blackburn, A. Piniella, and M. G. Cain, J. Appl. Phys. 103, 07 F506 (2008).

${ }^{15}$ Y. Zhang, L. Zheng, C. Deng, J. Ma, Y. Lin, and N. Ce-Wen, Appl. Phys. Lett. 92, 152510 (2008).

${ }^{16}$ E. Y. Tsymbal, A. Gruverman, V. Garcia, M. Bibes, and A. Barthélémy, MRS Bull. 37, 138-143 (2012).

${ }^{17}$ M. Gajek, M. Bibes, S. Fusil, K. Bouzehouane, J. Fontcuberta, A. Barthélémy, and A. Fert, Nat. Mater. 6, 296-302 (2007).

${ }^{18}$ R. Nechache, C. Harnagea, S. Licoccia, E. Traversa, A. Ruediger, A. Pignolet, and F. Rosei, Appl. Phys. Lett. 98, 202902 (2011).

${ }^{19}$ M. Vopson, Solid State Commun. 152, 2067-2070 (2012).

${ }^{20}$ C. W. Nan, M. I. Bichurin, S. Dong, D. Viehland, and G. Srinivasan, J. Appl. Phys. 103(3), 031101 (2008).

${ }^{21}$ Y. Wang, J. Hu, Y. Lu, and C.-W. Nan, NPG Asia Mater. 2(2) 61-68 (2010).

${ }^{22}$ J. Zhai, Z. Xing, S. Dong, J. Li, and D. Viehland, J. Am. Ceram. Soc. 91(2) 351-358 (2008).

${ }^{23}$ Y. Zhang, J. Liu, X. H. Xiao, T. C. Peng, C. Z. Jiang, Y. H. Lin, and C. W. Nan, J. Phys. D: Appl. Phys. 43, 082002 (2010).

${ }^{24}$ S. Sahoo, S. Polisetty, C.-g. Duan, S. S. Jaswal, E. Y. Tsymbal, and C. Binek, Phys. Rev. B 76, 092108 (2007).

${ }^{25}$ N. Moutis, D. S. Sandoval, and D. Niarchos, J. Magn. Magn. Mater. 320, 1050-1055 (2008).

${ }^{26}$ H. Boukari, C. Cavaco, W. Eyckmans, L. Lagae, and G. Borghs, J. Appl. Phys. 101, 054903 (2007).

${ }^{27}$ O. Kitakami, T. Shimatsu, S. Okamoto, Y. Shimada, and H. Aoi, Jpn. J. Appl. Phys., Part 2 43, L115-L117 (2004).

${ }^{28}$ D. Berlincourt and H. Jaffe, Phys. Rev. 111(1), 143-148 (1958).

${ }^{29}$ W. F. Brown, "Magnetic materials," in The Handbook of Chemistry and Physics, edited by E. U. Condon and H. Odishaw (McGraw-Hill, 1958), Chap. 8.

${ }^{30}$ E. Klokholm and J. A. Aboaf, J. Appl. Phys. 52(3), 2474-2476 (1981).

${ }^{31}$ M. D. Cooke, L.-C. Wang, R. Watts, R. Zuberek, G. Heydon, W. M. Rainforth, and G. A. Gehring, J. Phys. D: Appl. Phys. 33, 1450-1459 (2000).

${ }^{32}$ S. Dong, J. Zhai, J. Li, and D. Viehland, Appl. Phys. Lett. 89, 252904 (2006).

${ }^{33}$ T. L. Gilbert, Phys. Rev. 100, 1243 (1955).

${ }^{34}$ L. F. Sampine and M. K. Gordon, Computer Solution of Ordinary Differential Equations: The Initial Value Problem (Freeman, 1975).

${ }^{35}$ W. F. Brown, Jr., Micromagnetics (Krieger, New York, 1978).

${ }^{36}$ M. Vopsaroiu, M. Stewart, T. Hegarty, A. Muniz-Piniella, N. McCartney, M. Cain, and G. Srinivasan, Meas. Sci. Technol. 19, 045106 (2008).

${ }^{37}$ Y. Gao, J. M. Hu, L. Shu, and C. W Nan, Appl. Phys. Lett. 104(14), 142908 (2014). 